\title{
Health service usage and re-referral rates: comparison of a dietitian-first clinic with a medical specialist-first model of care in a cohort of gastroenterology patients
}

\author{
Rumbidzai Mutsekwa (1) , ${ }^{1,2}$ Szymon Ostrowski, ${ }^{3}$ Russell Canavan, ${ }^{3}$ \\ Lauren Ball, ${ }^{4,5}$ Rebecca Angus (i) 1,5
}

\begin{abstract}
${ }^{1}$ Nutrition Department, Gold Coast Hospital and Health Service, Southport, Queensland, Australia

${ }^{2}$ Centre for Applied Health Economics, Griffith University Faculty of Health, Gold Coast, Queensland, Australia ${ }^{3}$ Gastroenterology Department, Gold Coast Hospital and Health Service, Southport, Queensland, Australia

${ }^{4}$ Menzies Health Institute Queensland, Griffith University Faculty of Health, Gold Coast, Queensland, Australia

${ }^{5}$ School of Allied Health Sciences, Griffith University Faculty of Health, Gold Coast, Queensland, Australia
\end{abstract}

\section{Correspondence to}

Rumbidzai Mutsekwa, Nutrition Department, Gold Coast Hospital and Health Service, Southport, QLD 4215, Australia; rumbidzai. mutsekwa@health.qld.gov.au

Received 18 February 2020 Revised 10 April 2020

Accepted 19 April 2020

Published Online First

6 May 2020

\begin{abstract}
Background The dietitian-first gastroenterology clinic (DFGC) is an expanded scope of practice initiative implemented in response to increased gastroenterology specialist demand. This study examined re-referral rates to gastroenterology and overall health service usage up to 24 months post management in DFGC compared with a traditional, gastroenterology specialist-first model.

Methods Patients discharged from DFGC in the first year were matched with those seen in the traditional model. Demographic, clinical and process-related service characteristics were compared, and logistic regression analysis was undertaken to model re-presentation and model of care (MoC) as the variable of interest considering covariates in univariate analyses. Analyses were performed at 12, 18 and 24 months post discharge.
\end{abstract}

Results The DFGC (122 patients) and traditionalmodel (62 patients) cohorts had similar baseline demographic characteristics. Wait-times (68.6 vs 272.9 days; $p<0.001$ ), treatment-times (89.4 vs 259.9 days; $p<0.001)$ and usage of other services (1.4 vs 2.1 specialities; $p=0.01$ ) were lower in DFGC. Re-referral rates were low in both DFGC and traditional models at 12 months $(0.82 \%$ vs $1.61 \%), 18$ months ( $2.46 \%$ vs $6.45 \%)$ and 24 months ( $4.91 \%$ vs $8.06 \%)$, respectively, with no significant difference between the models at any time point.

Conclusion Most patients do not re-present for similar conditions within 2 years when managed in the DFGC or traditional medical model.

Patients managed in DFGC have lower overall health service usage compared with patients managed in the traditional model. These findings support the safety and effectiveness of a DFGC

\section{Summary box}

What is already known about this subject?

> There is increasing demand for gastroenterology medical specialist services with growing pressure on health service resources.

- Dietitians are well placed to work in extended scope of practice models of care to help address wait-list pressures in gastroenterology services, with reported high patient satisfaction levels, improved symptoms and quality of life for eligible patients.

What are the new findings?

> Patients seen in a dietitian-first gastroenterology clinic who present with altered bowel motions, abdominal pain, constipation, diarrhoea, dyspepsia/ heartburn/reflux, nausea or abdominal bloating and no alarm symptoms have low re-referral rates up to 24 months postdischarge similar to results seen in the traditional medical specialist-first model of care.

- Patients seen in the dietitian-first gastroenterology clinic have lower health service usage compared with patients seen in the traditional model of care.

model as one strategy to manage specialist gastroenterology service demands.

\section{INTRODUCTION}

High demand for medical specialist services has led to patients waiting longer than clinically recommended in many countries. $^{1} 2$ In Australia, the Health 
Summary box

\section{How might it impact on clinical practice in the} foreseeable future?

- These findings add to evidence that supports implementation of a dietitian-first gastroenterology clinic model as one strategy to help manage specialist gastroenterology service demands with demonstrated positive short-term and longer-term outcomes. This encourages continued discussion regarding incorporation of extended scope of practice roles within gastroenterology and other specialties to help improve the patient experience by providing better access and timely care for eligible patients.

Practitioner Expanded Scope of Practice programme was implemented to help reduce the number of patients waiting longer than clinically recommended, thereby improving patients' health journey and outcomes. ${ }^{3}$ This initiative extended the scope of allied health professionals beyond traditional limits. ${ }^{34}$ These models of care $(\mathrm{MoC})$ have been associated with reductions in medical specialist outpatient clinic waitlists and wait-times and have shown high levels of patient satisfaction while still providing safe and effective care for patients. ${ }^{4-6}$ In dietetics, extending scope of practice to include dysphagia screening, coeliac disease management, dietitian-first gastroenterology clinics (DFGC), postpyloric and gastrostomy tube placement has resulted in timely access to care for patients with potential cost savings. ${ }^{7-13}$

In our health service, the DFGC was implemented in June 2016 as one strategy to help reduce gastroenterology waitlists. This has reduced wait-times, so more patients are seen within clinically recommended timeframes. ${ }^{11}$ Furthermore, we have demonstrated positive short-term health outcomes of symptom severity reduction and improved quality of life. ${ }^{14}$ In this pathway, a consultant gastroenterologist screens referrals with patients aged between 18 and 50 years, presenting with altered bowel motions, abdominal pain, constipation, diarrhoea, dyspepsia/heartburn/reflux, nausea or abdominal bloating considered for triage to the DFGC. ${ }^{11}$ Patients are excluded if referral letter notes concerning (category 1) features as indicated in the Queensland Gastroenterology Clinical Prioritisation Criteria 2016. ${ }^{15}$ These features include weight loss $(\geq 5 \%$ of body weight in previous 6 months), iron deficiency in men and postmenopausal women, unexplained iron deficiency in premenopausal women, abnormal imaging, persistent abdominal pain, abdominal mass, gastrointestinal bleeding, nocturnal diarrhoea, persistent vomiting, dysphagia, history of polyps and/or personal or family history of Barrett's oesophagus, gastrointestinal cancers or inflammatory bowel disease, positive immunochemical faecal occult blood test and positive calprotectin. ${ }^{15}$ In the DFGC, dietitians working under the clinical governance of a gastroenterologist perform tasks that would typically be completed by a medical professional in traditional models. Trained dietitians screen for underlying pathology by assessing history of presenting illness, medical, family or social history, exclude red flags and have credentialing to order and interpret stool and blood tests. Where dietitian assessment identifies concerning features not indicated on the general practitioner (GP) referral, patients are expedited for a gastroenterology consultant review. ${ }^{11}$ Many low risk gastroenterology patients seen in the DFGC can be primarily managed by the dietitian before transfer of care back to their GP with satisfactory resolution of symptoms. Under the traditional model, gastroenterologists performed these tasks with few patients receiving dietetic input due to resource limitations. Figure 1 compares DFGC and traditional model pathways, including the flow of patients.

Comprehensive evaluations of new $\mathrm{MoC}$ must consider the long-term impact on health services and patient journey, including overall health service usage. Protracted wait-times for medical specialist care, dissatisfaction with health outcomes or decline in health condition after discharge from care may result in high resource utilisation and overall negative impact on patient physical and emotional well-being. ${ }^{16}$ Patient wait-times for the DFGC are lower than the traditional model, allowing earlier assessment and implementation of management strategies. However, it is unknown whether re-referral rates and number of gastroenterology and non-gastroenterology specialist appointments differ between patients seen in the DFGC and traditional MoC. Providing these objective measures of performance for new $\mathrm{MoC}$ is critical to ensure that patient care is timely and effective, safe and appropriate while supporting sustainability of healthcare systems.

The primary aim of this study was to determine the re-referral rates to gastroenterology specialist outpatient services or presentations to emergency departments with similar complaints for patients who had been managed and discharged from the DFGC when compared with similar patients managed in the traditional, gastroenterologist-first MoC. The second aim was to examine the overall healthcare resource usage of patients managed in each model by determining patient activity within gastroenterology and other public specialist medical services.

\section{METHODS}

\section{Study design}

This was a single-centre retrospective comparison audit in a tertiary gastroenterology service in Queensland, Australia.

\section{Participants and setting}

All patients discharged from the DFGC in the initial 12 months of operation (22 June 2016-21 June 2017) were criteria matched with those seen under the previous traditional medical-first model from 22 June 


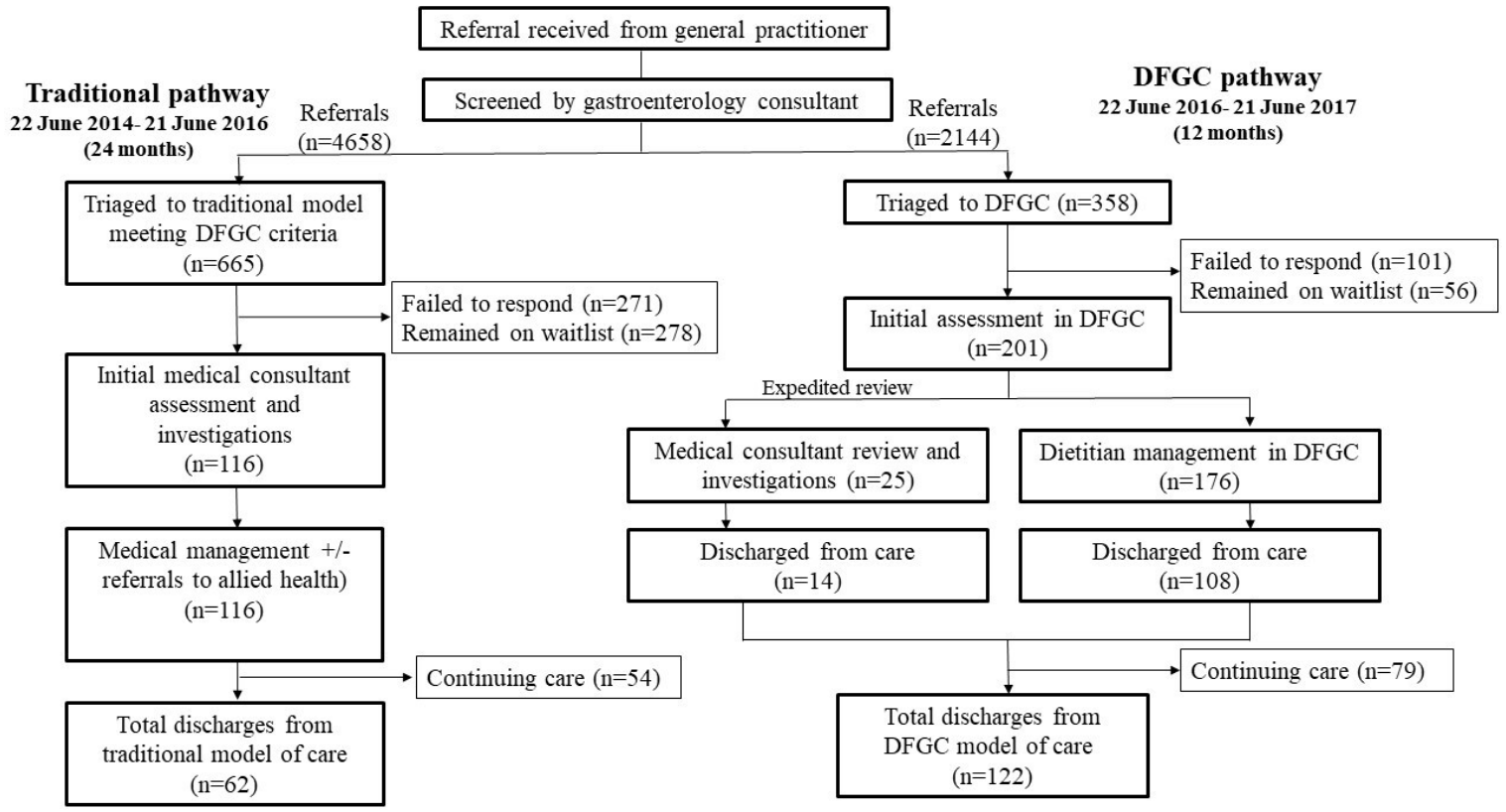

Figure 1 Flow diagram comparing traditional gastroenterology medical pathway and DFGC pathways. This illustration also shows the flow of patients before (traditional model) and after implementation of the DFGC. DFGC, dietitian-first gastroenterology clinic.

201421 June 2016. Matching was based on the DFGC selection criteria described, including age, presenting condition, clinical categorisation and absence of concerning features. $^{15}$

\section{Data collection}

Electronic medical records were reviewed to extract original referrals for included patients. New referrals to all state-wide public gastroenterology specialist services with electronic medical records were identified, as were gastroenterology related presentations to emergency departments within 24 months of discharge from either MoC. To be classified as a re-referral, new referrals outlined the same condition as the original or included a statement of re-referral to access gastroenterology specialist services. Data on re-referrals were extracted in duplicate by two clinicians with findings compared and any inconsistencies decided through discussion. Information on patient service usage including number of appointments within gastroenterology services and patient activity with other medical specialities up to 2 years postdischarge was captured. The referral date, discharge date, reason for referral, triage category, patient wait-time (referral to first appointment), treatmenttime (first appointment to discharge) and length of episode of care (referral to discharge) associated with the first referral were recorded, along with demographic information including age, gender, smoking status and presence of other comorbidities including anxiety and depression.

\section{Data analysis}

Prior to analysis, a data entry accuracy check of $10 \%$ against source data was conducted by a second investigator. Histograms were used to check data normality, and level of significance was set at $\mathrm{p}<0.05$. Stata (V.15.1, Stata, College Station, Texas, USA) was used to examine differences in distributions of gender, referral reason and smoking status (Pearson's $\chi^{2}$ and Fisher's exact tests), mean age, weight and body mass index (paired t-tests), median wait-times, treatmenttimes and length of episode of care (Mann-Whitney U tests). Logistic regression analysis was undertaken to model re-referral with $\mathrm{MoC}$ as the variable of interest and considered the covariates noted above in univariate analyses. Analyses were performed at 12, 18 and 24 months after discharge from each MoC.

\section{RESULTS}

A total of 122 patients were discharged from the DFGC in the study period. Fourteen (11.4\%) patients had been expedited for gastroenterology review after concerning features were identified by the dietitian during initial assessment. In the traditional $\mathrm{MoC}$, 62 patients were seen and discharged in the 2 years preceding establishment of the DFGC (figure 1). Patients were similar in each MoC, with the only significant difference being a lower percentage of patients in DFGC having diarrhoea or constipation as referral reason (table 1). Patients were predominantly female with a substantial proportion reporting anxiety or depression.

Patients seen in the DFGC have significantly shorter wait-times (68.6 vs 272.9 days, $\mathrm{p}<0.001)$, treatment times (89.4 vs 259.9 days, $\mathrm{p}<0.001)$ and total episode of care lengths $(177.5$ days vs 532.5 days, $\mathrm{p}<0.001)$ compared with those in the traditional MoC. The average number of appointments required by patients 


\begin{tabular}{|c|c|c|c|}
\hline & $\begin{array}{l}\text { DFGC } \\
(n=122)\end{array}$ & $\begin{array}{l}\text { Traditional medical model } \\
(\mathrm{n}=62)\end{array}$ & P value \\
\hline Age, years & $30.9 \pm 7.7(18-49)$ & $31.7 \pm 9.2(18-50)$ & 0.52 \\
\hline Women, n (\%) & $89(73.0)$ & $49(79.0)$ & 0.37 \\
\hline Men, n (\%) & $33(27.0)$ & $13(21.0)$ & \\
\hline \multicolumn{4}{|l|}{ Smoking status } \\
\hline Current smokers, $\mathrm{n}(\%)$ & $31(23.2)$ & $17(27.4)$ & 0.97 \\
\hline Ex-smokers, n (\%) & $22(18.0)$ & $6(9.7)$ & \\
\hline Non-smokers, n (\%) & $69(56.6)$ & $38(61.3)$ & \\
\hline Missing data, $\mathrm{n}(\%)$ & $0(0)$ & $1(1.6)$ & \\
\hline Weight, kg & $79.0 \pm 21.4(42-205)$ & $81.3 .0 \pm 21.7(45-165)$ & 0.5 \\
\hline $\mathrm{BMI}, \mathrm{kg} / \mathrm{m}^{2}$ & $27.4 \pm 7.0(17-70)$ & $29.3 \pm 7.3(18-54)$ & 0.12 \\
\hline Underweight, $<18.5, \mathrm{n}(\%)$ & $3(2.5)$ & $1(1.6)$ & \\
\hline Healthy weight, 18.5-24.99, n (\%) & $47(38.5)$ & $9(14.5)$ & \\
\hline Overweight 25-29.99, n (\%) & $38(31.1)$ & $21(33.9)$ & \\
\hline Obese, $>30, n(\%)$ & $33(27.0)$ & $16(14.8)$ & \\
\hline Missing data $\mathrm{n}(\%)$ & $1(0.8)$ & $15(13.9)$ & \\
\hline \multicolumn{4}{|l|}{ Referral reason based on GP letter, ${ }^{*} \mathrm{n}(\%)$} \\
\hline Abdominal pain & $62(50.8)$ & $35(56.5)$ & 0.35 \\
\hline Altered bowel habit & $8(6.6)$ & $2(3.2)$ & 0.53 \\
\hline Constipation & $19(15.6)$ & $20(32.3)$ & 0.01 \\
\hline Diarrhoea & $44(36.1)$ & $29(46.8)$ & 0.03 \\
\hline Dyspepsia/Heartburn/Reflux & $21(17.2)$ & $5(8.1)$ & 0.13 \\
\hline Nausea & $11(9.0)$ & $9(14.5)$ & 0.54 \\
\hline Bloating & $31(25.4)$ & $15(24.2)$ & 0.71 \\
\hline \multicolumn{4}{|l|}{ Anxiety/Depression, n (\%) } \\
\hline Nil reported/Nil diagnosis & $64(52.5)$ & $42(67.7)$ & 0.12 \\
\hline Medically diagnosed & $45(36.9)$ & $18(29.0)$ & \\
\hline Self-reported & $13(10.7)$ & $2(3.2)$ & \\
\hline
\end{tabular}

*Some patients had more than one presenting complaint; hence referral reasons total is greater than $>100 \%$.

$\mathrm{BMI}$, body mass index; DFGC, dietitian-first gastroenterology clinic; MoC, model of care.

did not differ between the two MoC (DFGC 2.4 \pm SD 1.34 , range $1-9$ vs traditional model $2.4 \pm$ SD 1.34 , range $1-7 ; p=0.99)$. Health service usage was lower for patients seen in the DFGC, who were under the care of fewer additional medical specialities within our health service in comparison to those in the traditional MoC $(1.37 \pm$ SD 1.78 , range $0-8$ vs $2.1 \pm$ SD 2.08, range $0-9 ; \mathrm{p}=0.01)$.

Ten new gastroenterology referrals were received for patients previously managed in the DFGC within
24 months of their discharge. Of these, six referrals were for the same conditions previously managed, with an average re-referral time of $533 \pm$ SD 133 days (range 346-691). In the traditional $\mathrm{MoC}$, there were nine new referrals with five deemed to be re-referrals. The average re-referral time was $496 \pm$ SD 149 days (range 333-719). Representation rates for both MoC were low, with no statistically significant differences between these at either 12,18 or 24 months postdischarge (table 2). The reasons for re-referral were

Table 2 Number of patients re-referred for similar complaints in the DFGC vs the traditional MoC, within 12, 18 and 24 months after discharge

\begin{tabular}{|c|c|c|c|c|c|}
\hline Re-referral period & $\begin{array}{l}\text { DFGC } \\
(n=122)\end{array}$ & $\begin{array}{l}\text { Traditional model } \\
(\mathrm{n}=62)\end{array}$ & $\begin{array}{l}\text { OR } \\
\text { (DFGC/TM) }\end{array}$ & $95 \% \mathrm{Cl}$ & $P$ value \\
\hline $\begin{array}{l}\text { Re-referrals at } 12 \text { months } \\
n(\%)\end{array}$ & $1(0.82)$ & $1(1.61)$ & 0.51 & 0.03 to 8.33 & 0.64 \\
\hline $\begin{array}{l}\text { Re-referrals at } 18 \text { months } \\
\text { n (\%) }\end{array}$ & $3(2.46)$ & $4(6.45)$ & 0.37 & 0.07 to 1.68 & 0.20 \\
\hline $\begin{array}{l}\text { Re-referrals at } 24 \text { months } \\
\text { n (\%) }\end{array}$ & $6(4.91)$ & $5(8.06)$ & 0.55 & 0.16 to 1.19 & 0.33 \\
\hline
\end{tabular}

DFGC, dietitian-first gastroenterology clinic; MoC, model of care. 
similar in the DFGC and traditional MoC. Each had two patients re-referred for abdominal pain and one for recurrent diarrhoea. The DFGC had three patients re-referred for gastroesophageal reflux disease (GERD) or epigastric pain, while the traditional model had two such re-referrals.

At the time of audit, 9 of the 11 patients re-referred after care in either $\mathrm{MoC}$ had been reassessed by a gastroenterology consultant and had endoscopic or imaging investigations. The remainder failed to attend appointments and were discharged from the service. Of note, initial management strategies did not change after further assessment and investigations.

\section{DISCUSSION}

In gastroenterology, models of care that use expanded scope of practice for non-medical practitioners show promise in providing high quality and safe care while reducing wait-time and waitlists for eligible patients. ${ }^{11} 12$ Comprehensive evaluations should also consider whether health improvements are sustained over a longer period. One method to assess this is examination of the rate of patient re-referral with similar complaints. ${ }^{17}$ The research presented here adds evidence for the long-term benefit of extended scope of practice roles in allied health on the patient journey and healthcare resources.

In this study, we found that re-referrals of patients discharged from the DFGC were very low, with less than $5 \%$ of patients re-referred with similar complaints within 24 months. This was lower although not statistically different from the traditional model where the re-referral rate was approximately $8 \%$. The main reason for re-referral was deterioration or unsatisfactory further symptom improvement after discharge. Concerning features had not been overlooked in either MoC, with no missed or alternate diagnoses identified when records were reviewed. Management strategies recommended to re-presenting patients were not substantially different from guidance provided in initial assessments. These findings support the safety and effectiveness of dietitian-first gastroenterology $\mathrm{MoC}$ with re-referrals comparable to those in a traditional medical first $\mathrm{MoC}$ for selected patients.

Many patients meeting the criteria for the DFGC have functional gastrointestinal disorders (FGIDs). In patients unlikely to have life-threatening pathology, diagnostic tests do not reassure and can lead to further healthcare-seeking behaviour in search for an organic cause for symptoms. ${ }^{18}$ These patients have higher number of hospitalisations, surgical procedures, primary care visits and prescriptions compared with patients without FGIDs. ${ }^{18} 19$ Many patients with FGIDs have regular visits to medical specialists for both gastrointestinal and non-gastrointestinal symptoms with repeat consultation often leading to greater number or overuse of investigations. ${ }^{18}$ Our findings were consistent with this, with some patients under the care of up to nine other medical specialities. However, it was notable that patients managed in the DFGC had significantly less referrals and appointments with other medical specialists compared with patients managed in the traditional MoC.

To develop strategies for effective patient management, an understanding of the factors associated with high overall health service usage is required. These include healthcare factors (eg, suboptimal health and social care), patient-related factors (eg, social and family environment, treatment adherence) and diseaserelated factors (eg, comorbidities, natural progression of medical conditions). ${ }^{20}$ Re-referrals for conditions managed previously may reflect on the effectiveness of patient management and has implications on cost efficiency. High re-referral rates also have negative impacts on waitlists implying prolonged periods of continued unmet needs for patients. A significant proportion of patients seen under either $\mathrm{MoC}$ reported a medical or self-diagnosis of anxiety or depression. The FGIDs are a result of complex interactions between biological, psychological and social factors and can only be treated satisfactorily when all these factors are considered and addressed. Formal psychological screening and management addressing psychological variables is therefore imperative as it impacts both health related quality of life and healthcare use and thus medical and societal cost. ${ }^{21}$

This study indicates that dietitians working in an expanded scope of practice with clinical guidance from a consultant gastroenterologist can provide an early and clear diagnosis in this selected cohort of patients. Explanations regarding the chronic and often recurrent nature of conditions, with management options focused on the reduction of bothersome symptoms and the prevention of dietary and other lifestyle triggers are of benefit to patients. It is important to provide patients with an early diagnosis using clear language along with associated management strategies to help reduce the amount of low yield testing and re-referrals to medical care with ongoing symptoms, ${ }^{22}$ and this has been a key focus for the DFGC MoC.

Interpretation of findings should consider study strengths, limitations and generalisability to other settings. The implementation of the DFGC in our service resulted in a change of practice; thus, MoC could not be compared concurrently and we cannot discount possible influence of outside confounders related to cohorts being derived from different periods of time. Retrospective studies may have significant biases related to selection of controls. We have mitigated this risk by including all patients seen in given time periods who met DFGC eligibility criteria. Our criteria matching provided cohorts with similar baseline characteristics, suggesting this process provided a valid comparator. Retrospective studies often require larger samples sizes to detect rare outcomes which may have limited our ability to detect significant difference 
in re-referral rates between MoC. New referrals to all public gastroenterology services with integrated electronic medical records state-wide were included, as were emergency department presentations for similar complaints to the patient's original referral. However, information from health services without electronic medical records, and from private providers could not be accessed. Integration of health records across public health services, primary care and the private sector may allow this to be addressed in future studies.

This model, where eligible patients are screened and redirected by the consultant gastroenterologist from waitlists to the DFGC for management by a trained dietitian, has resulted in high patient satisfaction, positive short-term patient health outcomes and positive process related outcomes which allow gastroenterologists to see more complex and urgent cases. ${ }^{11}{ }^{14}$ In this study, we have shown that improvements seen after management in the DFGC can be sustained over a long period of time, demonstrated by very low referral rates up to 24 months after discharge which were lower, although not statistically different, to traditional model re-presentation rates. Few studies have examined the cost benefits of extended scope of practice roles when compared with physician led MoC. Comprehensive short-term and long-term economic impacts must be examined to determine MoC sustainability and should consider cost benefits associated with low re-presentation rates, such as that seen for DFGC patients.

In summary, we found that in our service patients are not re-referred for similar conditions within 2 years when managed in either the DFGC or traditional medical model (95\% and 92\%, respectively). We have also described public health system service usage in this patient group which adds to the literature on how gastroenterology patients use health resources. These findings further support the safety, quality and effectiveness of a DFGC model as a strategy to manage specialist gastroenterology service demands and support continued discussion regarding incorporation of extended scope of practice roles within gastroenterology and other specialties to help improve the patient experience by providing better access and timely care for eligible patients.

\section{Twitter Russell Canavan@CanavanRussell}

Acknowledgements The authors acknowledge the Gold Coast Hospital and Health Service for funding this model of care. They thank the staff of the GCHHS gastroenterology, nutrition and data management departments for their support and Ian Hughes for his assistance with statistical analysis.

Contributors RC, RM, LB and RA devised the project devised the project, the main conceptual ideas and proof outline. RM and SO collated, analysed and interpreted the data and drafted the manuscript. All authors read and approved the final manuscript.

Funding This work was supported by the Allied Health Profession Office of Queensland (AHPOQ) Health Practitioner Research Scheme (grant number AH001649). Lauren Ball is supported by an NHMRC Investigator Grant (APP1173496).
Competing interests None declared.

Patient consent for publication Not required.

Ethics approval This study was approved by the Gold Coast Hospital and Health Service Human Research Ethics Committee (HREC Reference: HREC/18/QGC/56).

Provenance and peer review Not commissioned; externally peer reviewed.

Data availability statement All data relevant to the study are included in the article or uploaded as supplementary information. However, further information can be obtained from the corresponding author.

\section{ORCID iDs}

Rumbidzai Mutsekwa http://orcid.org/0000-0001-8200-3026

Rebecca Angus http://orcid.org/0000-0002-1839-9641

\section{REFERENCES}

1 Siciliani L, Hurst J. Tackling excessive waiting times for elective surgery: a comparative analysis of policies in 12 OECD countries. Health Policy 2005;72:201-15.

2 Segal L, Bolton T. Issues facing the future health care workforce: the importance of demand modelling. Aust New Zealand Health Policy 2009;6:12.

3 Allied Health Professions' Office of Queensland. Ministerial Taskforce on health practitioner expanded scope of practice: final report. Brisbane: Queensland government 2014.

4 Stute M, Moretto N, Raymer M, et al. Process to establish 11 primary contact allied health pathways in a public health service. Aust Health Rev 2017.

5 McPherson K, Kersten P, George S, et al. A systematic review of evidence about extended roles for allied health professionals. J Health Serv Res Policy 2006;11:240-7.

6 Saxon RL, Gray MA, Oprescu FI. Extended roles for allied health professionals: an updated systematic review of the evidence. J Multidiscip Healthc 2014;7:479-88.

7 Porter J. Transdisciplinary screening and intervention for nutrition, swallowing, cognition and communication: a case study. J Res Interprof Pract Educ 2014;4.

8 Rollins CM. Blind bedside placement of postpyloric feeding tubes by registered dietitians: success rates, outcomes, and cost effectiveness. Nutr Clin Pract 2013;28:506-9.

9 Simmance N, Cortinovis T, Green C, et al. Introducing novel advanced practice roles into the health workforce: dietitians leading in gastrostomy management. Nutr Diet 2019;76:1420.

10 Stuckey C. Management of adult coeliac disease with a dietitian-led virtual clinic. Gastrointestinal Nursing 2015;13:38-43.

11 Mutsekwa RN, Canavan R, Whitfield A, et al. Dietitian first gastroenterology clinic: an initiative to reduce wait Lists and wait times for gastroenterology outpatients in a tertiary hospital service. Frontline Gastroenterol 2019;10:229-35.

12 Ryan D, Pelly F, Purcell E. The activities of a dietitian-led gastroenterology clinic using extended scope of practice. $B M C$ Health Serv Res 2016;16:604.

13 Ryan D, Pelly F, Purcell E. Exploring extended scope of practice in dietetics: a systems approach. Nutr Diet 2017;74:334-40.

14 Mutsekwa RN, Larkins V, Canavan R, et al. A dietitian-first gastroenterology clinic results in improved symptoms and quality of life in patients referred to a tertiary gastroenterology service. Clin Nutr ESPEN 2019;33:188-94.

15 Queenland Health Clinical Excellence Division. Clinical prioritisation criteria. Gastroenterology 2016.

16 Dunnill MGS, Pounder RE. Medical outpatients: changes that can benefit patients. Clin Med 2004;4:45-9.

17 Chang AT, Gavaghan B, O'Leary S, et al. Do patients discharged from advanced practice physiotherapy-led clinics 
re-present to specialist medical services? Aust Health Rev 2018;42:334-9.

18 Drossman DA, Li Z, Leserman J, et al. Health status by gastrointestinal diagnosis and abuse history. Gastroenterology 1996;110:999-1007.

19 El-Serag HB, Olden K, Bjorkman D. Health-Related quality of life among persons with irritable bowel syndrome: a systematic review. Aliment Pharmacol Ther 2002;16:1171-85.

20 Lyratzopoulos G, Havely D, Gemmell I, et al. Factors influencing emergency medical readmission risk in a UK district general Hospital: a prospective study. BMC Emerg Med 2005;5:1.

21 Levy RL, Olden KW, Naliboff BD, et al. Psychosocial aspects of the functional gastrointestinal disorders. Gastroenterology 2006;130:1447-58.

22 Linedale EC, Chur-Hansen A, Mikocka-Walus A, et al. Uncertain Diagnostic Language Affects Further Studies, Endoscopies, and Repeat Consultations for Patients With Functional Gastrointestinal Disorders. Clin Gastroenterol Hepatol 2016;14:1735-41. 